\title{
An alternative synthetic route to the neuroleptic compound Pipothiazine
}

\author{
Gabriela P. Sarmiento, ${ }^{a}$ Graciela Y. Moltrasio, ${ }^{a}$ and Albertina G. Moglioni ${ }^{b}$ * \\ ${ }^{a}$ Cátedra de Química Orgánica III, ${ }^{b}$ Cátedra de Química Medicinal, \\ Facultad de Farmacia y Bioquímica. Universidad de Buenos Aires \\ Junín 956 (1113) Buenos Aires, Argentina \\ E-mail:amoglio@ffyb.uba.ar
}

\begin{abstract}
A simple and efficient synthesis of the neuroleptic compound Pipothiazine $\mathbf{1}$ is described, involving the reductive cyclation of (2-nitrophenyl)(phenyl)sulfanes $\mathbf{3}$ for the formation of the phenothiazine ring.
\end{abstract}

Keywords: Phenothiazine, molecular rearrangement, nitrene, microwave irradiation, borane

\section{Introduction}

Phenothiazines were the first agents successfully used for the treatment of psychosis; at present, they are widely known for their clinical use as antipsychotic, antiemetic, antihypertensive, and antihistaminic agents, among other therapeutic uses. ${ }^{1}$ In recent years, phenothiazines proved to act as human cholinesterase inhibitors, ${ }^{2}$ and on many occasions, they have been characterized as multidrug resistance (MDR) reversal agents. ${ }^{3}$ Neuroleptic phenothiazines have been used for over fifty years, and several methods for the preparation of these compounds have been reported, mostly in patents of pharmaceutical companies, as for example Pipothiazine $1{ }^{4}$

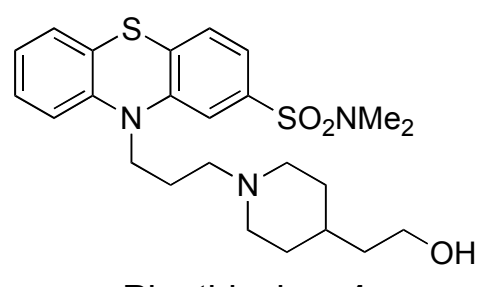

Pipothiazine, 1

Some methods for the synthesis of phenothiazines are well known. Phenothiazines with substituents in position 2, 3, and 4 can be obtained via [2-[(2-chlorophenyl)thio]phenyl]amine 
derivatives. ${ }^{3}$ A commonly used method for the preparation of 2-substituted phenothiazines is the reaction of 2-aminobenzenethiol with derivatives of 2-chloro-1-nitrobenzene followed by acetylation and Smiles rearrangement. ${ }^{5,6}$

This paper describes an alternative synthetic route to Pipothiazine $\mathbf{1}$ by preparing an appropriately substituted phenothiazine followed by aminoalkylation at nitrogen (position 10).

\section{Results and Discussion}

\section{Preparation of the phenothiazine ring}

For the preparation of the phenothiazine ring in $\mathbf{4}$ we considered (2-nitrophenyl)(phenyl)sulfane 3 to undergo cyclization via a nitrene intermediate. ${ }^{7,8}$

The reaction of benzenethiol and $o$-chloronitrobenzene (2a) in tetrahydrofuran in the presence of sodium hydride furnished (2-nitrophenyl)(phenyl)sulfane (3a), which upon treatment with triethyl phosphite in cumene ${ }^{9}$ rendered phenothiazine (4a) (Scheme 1). The reductive cyclation of (2-nitrophenyl)(phenyl)sulfane (3a) with ethyl triphosphite reduces the number of steps and furnishes compound $\mathbf{4 a}$ in good yield. Products $\mathbf{3 a}{ }^{10}$ and $\mathbf{4} \mathbf{a}^{11}$ were characterized by spectroscopic and physical data in accordance with literature data.

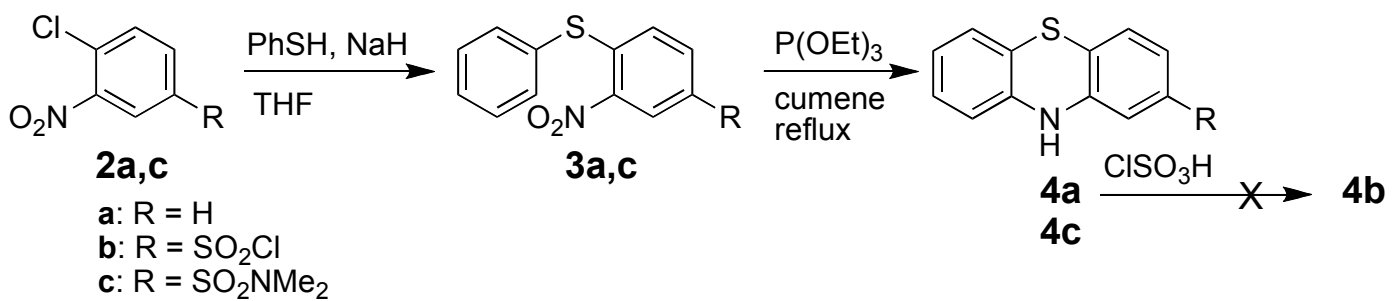

\section{Scheme 1}

The sulfonation of phenothiazine (4a) with chlorosulfonic acid failed to afford the desired $10 \mathrm{H}$-phenothiazine-2-sulfonyl chloride (4b), as indicated by ${ }^{1} \mathrm{H}$ NMR spectra of the reaction product. According to the literature, the electrophilic aromatic substitution of the phenothiazine ring is difficult because of polysubstitution and facile oxidation of the sulfur atom. ${ }^{12}$

This prompted an alternative approach employing an appropriately substituted benzenethiol and $o$-chloronitrobenzene as the starting materials. Sulfonation of benzenethiol with concentrated sulfuric acid at room temperature or at $100{ }^{\circ} \mathrm{C}$ yielded a mixture of mercaptobenzenesulfonic acid regioisomers; the desired para-isomer could not be isolated as a pure product.

Chlorosulfonation of $o$-chloronitrobenzene (2a) was tested under several conditions; the best procedure is addition of chlorosulfonic acid to $o$-chloronitrobenzene (2a) and heating the mixture to $130{ }^{\circ} \mathrm{C}$ for $30 \mathrm{~h}$. The resulting product was a solid compound exhibiting a ${ }^{1} \mathrm{H}$ NMR spectrum in agreement with the structure of 4-chloro-3-nitrobenzene-1-sulfonyl chloride (2b) (Scheme 2). When sulfonyl chloride $\mathbf{2 b}$ was treated with dimethylamine in ethanol, a solid product was 
obtained: 4-chloro- $N, N$-dimethyl-3-nitrobenzenesulfonamide (2c) ${ }^{13}$ along with a small amount of 4-(dimethylamino)- $N, N$-dimethyl-3-nitrobenzenesulfonamide (5) (Scheme 2). Both products 2c and 5 were separated by chromatography and characterized.

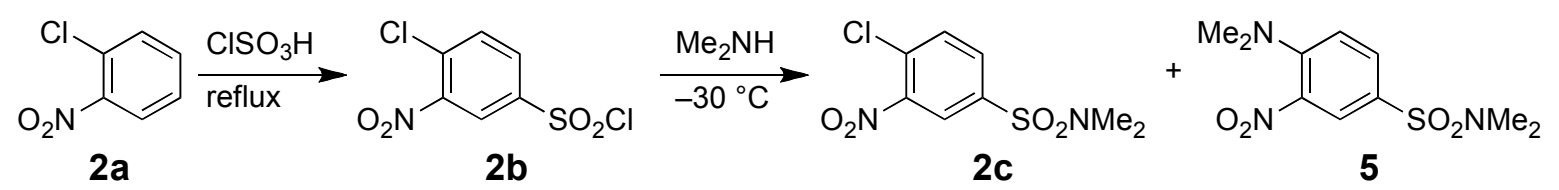

\section{Scheme 2}

The nucleophilic displacement of the chlorine atom in 4-chloro- $N, N$-dimethyl-3-nitrobenzenesulfonamide (2c) by benzenethiol rendered (2-nitrophenyl)(phenyl)sulfane $(\mathbf{3 c})^{14}$ as a solid product (Scheme 1). Thioether 3c has been previously obtained from the same reactants under different reaction conditions, but neither yield nor spectroscopic data have been reported. ${ }^{14}$ For the formation of the phenothiazine ring, the reductive cyclization of (2-nitrophenyl)(phenyl)sulfane (3b) was performed (Scheme 1). Such a procedure involving a nitrene intermediate has been described for the synthesis of substituted phenothiazines, ${ }^{9}$ but has not been applied to the preparation of $N, N$-dimethyl-10H-phenothiazine-2-sulfonamide (4c). Structure $4 \mathbf{c}$ was assigned from spectroscopic data. Compound $\mathbf{4 c}$ has been synthesized previously ${ }^{15,16}$ from substituted (2-nitrophenyl)(phenyl)sulfanes $\mathbf{3}$ by different procedures involving more steps.

\section{Attachment of the aminoalkyl chain at the nitrogen atom of the phenothiazine ring}

First, we studied the incorporation of a 3-carbon chain into 2-(piperidin-4-yl)ethanol (6), a commercially available compound, to obtain 3-(4-(2-hydroxyethyl)piperidin-1-yl)-3-oxopropanoic acid (7) (Scheme 3). This would enable the attachment of the aliphatic substituent at the nitrogen in position 10 of phenothiazine $4 \mathbf{c}$ to form 10-[3-[4-(2-hydroxyethyl)piperidin-1-yl]3-oxopropanoyl]- $N, N$-dimethyl-10H-phenothiazine-2-sulfonamide (8).

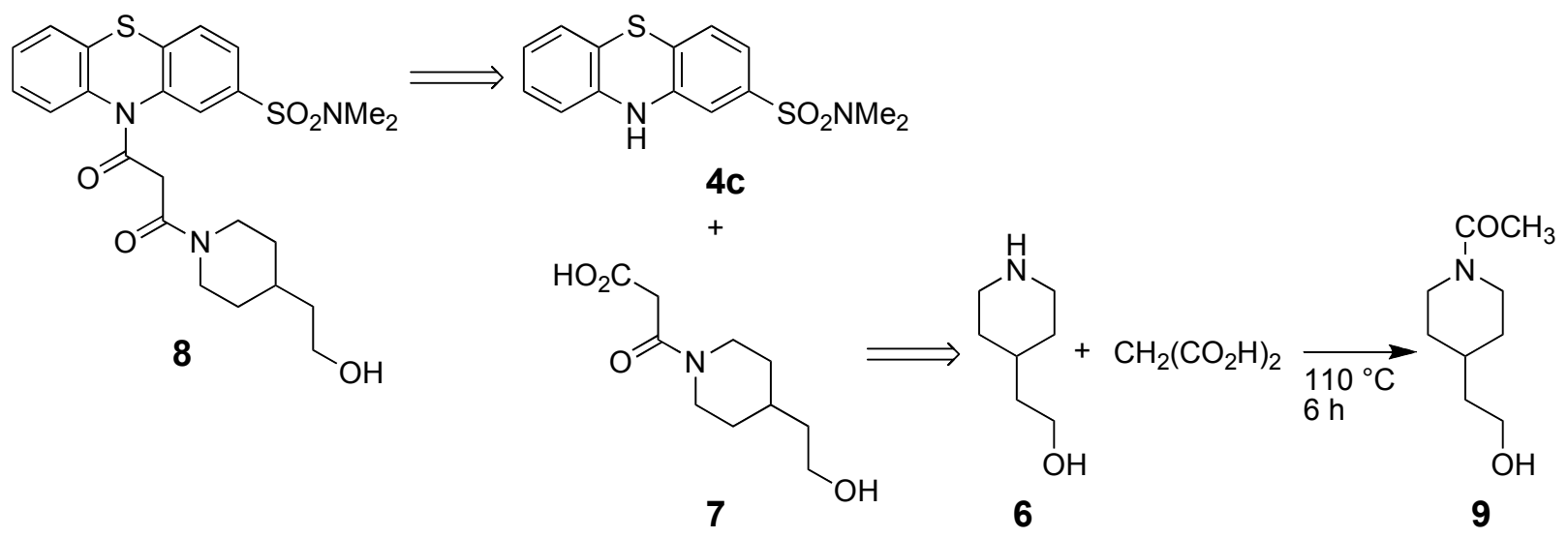

Scheme 3 
To this end, piperidine 6 was heated with malonic acid but the reaction did not afford the desired amide 7; instead, the $\mathrm{N}$-acetyl derivative 9 was obtained.

Since amide 8 could not be prepared by this approach, the amidation of the unsubstituted phenothiazine 4a with $\beta$-chloropropanoyl chloride was studied. Amide $\mathbf{1 0 a}^{17}$ was easily obtained both by conventional conditions and by microwave irradiation; the latter method increased the yield to $95 \%$ within a reaction time of 7 minutes. Subsequent substitution of the chlorine atom in 10a with 2-(piperidin-4-yl)ethanol (6) afforded product 11a (Scheme 4).

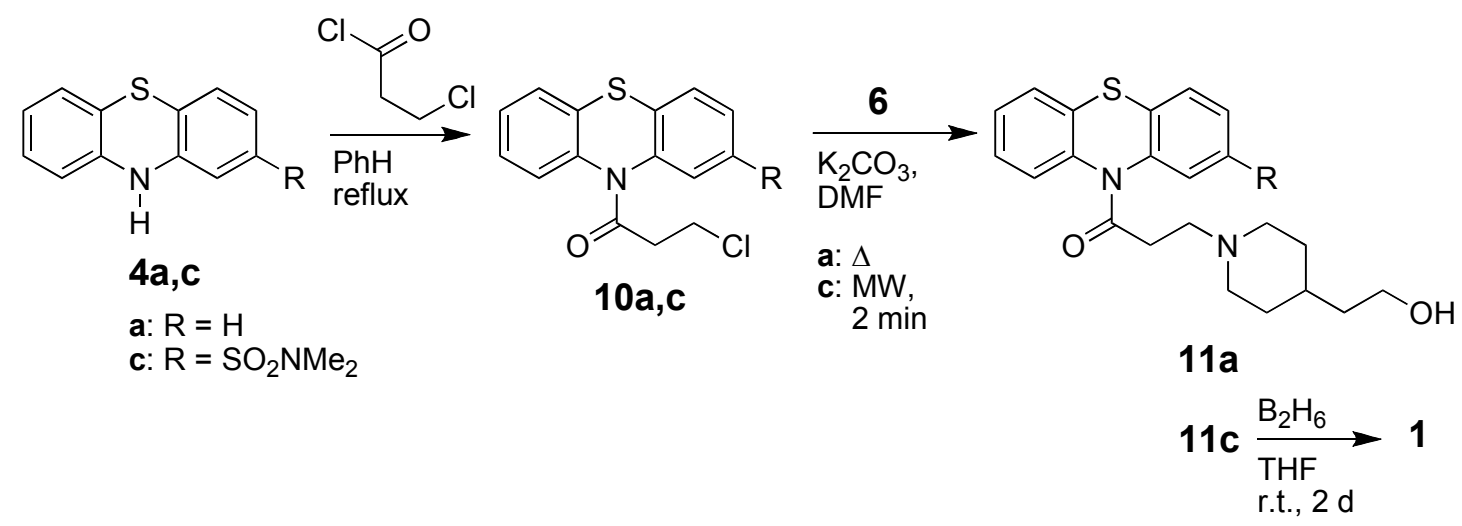

\section{Scheme 4}

Applying these conditions to phenothiazine $4 \mathbf{c}$, the reaction with $\beta$-chloropropanoyl chloride at reflux temperature gave compound $10 \mathbf{c}^{15}(62 \%)$. Subsequent reaction with 2-(piperidin-4yl)ethanol (6) converted 10c into 11c; microwave irradiation provided 11c quantitatively within a few minutes.

As known from the literature ${ }^{12}$ the reduction of the carbonyl group using lithium aluminum hydride is not appropriate for acyl derivatives of phenothiazine. Therefore, product 11c was reduced with diborane in tetrahydrofuran to form Pipothiazine 1 (60\%). The spectroscopic data were identical with those obtained from an authentic sample.

\section{Conclusions}

We have developed a simple method for the synthesis of the neuroleptic compound Pipothiazine, starting from commercially available reagents.

\section{Experimental Section}

General Procedures. The commercial reagents were used without purification. Melting points were determined with a Thomas-Hoover apparatus. Reaction progress was monitored by TLC 
(aluminum sheets coated with Merck silica gel $\mathrm{GF}_{254}$ ). Silica gel was used for flash and column chromatography (Merck silica gel 60, 230-400 mesh) and for preparative TLC (Merck silica gel $\left.60 \mathrm{GF}_{254}\right)$. Microwave irradiation was performed with a domestic oven $(150 \mathrm{~W})$. IR spectra were recorded using a Perkin-Elmer Spectrum One FT-IR spectrophotometer. NMR spectra were recorded on Bruker Avance II 500, Bruker Avance II 400 or Bruker AC 300 spectrometers. Mass spectra (MALDI/DCTB, APCI, ESI) were recorded on a Shimadzu QP 5000 spectrometer. Microanalyses were performed by Universidad Nacional de la Plata (La Plata-Argentina) on a Carlo Erba elemental analyzer 1106.

4-Chloro-3-nitrobenzensulfonyl chloride (2b). Chlorosulfonic acid (12.7 mL, $191.30 \mathrm{mmol})$ was added dropwise to 4-chloro-3-nitrobenzene (2a; $10 \mathrm{~g}, 63.50 \mathrm{mmol})$, and the mixture was stirred and heated at $130{ }^{\circ} \mathrm{C}$ for $30 \mathrm{~h}$. The reaction mixture was poured on ice, the resulting offwhite solid was filtered off and purified by column chromatography (dichloromethane/hexane, 1:1) to give $2 \mathrm{~b}(9.7 \mathrm{~g}, 60 \%)$; mp 57-60 ${ }^{\circ} \mathrm{C}$. $\mathrm{R}_{f}=0.56$ (dichloromethane/ hexane, 1:1). IR (KBr): v 1546, 1388, 1364, 1182, 1049, 901, 830, $775 \mathrm{~cm}^{-1} .{ }^{1} \mathrm{H}$ NMR $\left(500 \mathrm{MHz}, \mathrm{CDCl}_{3}\right): \delta 7.87\left(\mathrm{~d},{ }^{3} J\right.$ $=8.5 \mathrm{~Hz}, 1 \mathrm{H}, \mathrm{H}-5), 8.18\left(\mathrm{dd},{ }^{3} J=8.2 \mathrm{~Hz},{ }^{4} J=1.8 \mathrm{~Hz}, 1 \mathrm{H}, \mathrm{H}-6\right), 8.55\left(\mathrm{~d},{ }^{4} J=2.2 \mathrm{~Hz}, 1 \mathrm{H}, \mathrm{H}-2\right)$. ${ }^{13} \mathrm{C}$ NMR (125 MHz, $\mathrm{CDCl}_{3}$ ): $\delta$ 124.3, 130.5 (2C), 133.7, 134.6, 143.1. MS (MALDI/DCTB): $m / z(\%) 219.9(100)[\mathrm{M}-\mathrm{Cl}]^{+}$.

4-Chloro- $N, N$-dimethyl-3-nitrobenzenesulfonamide $(2 c)^{13}$ and 4-dimethylamino- $N, N$ dimethyl-3-nitrobenzenesulfonamide (5). A $33 \%$ solution of $N, N$-dimethylamine in absolute ethanol $(1.6 \mathrm{~mL}, 11.70 \mathrm{mmol})$ was added dropwise to a solution of 4-chloro-3-nitrobenzenesulfonyl chloride (2b) $(3.00 \mathrm{~g}, 11.70 \mathrm{mmol})$ in dichloromethane $(5 \mathrm{~mL})$ at $-30{ }^{\circ} \mathrm{C}$, followed by addition of $50 \%$ aq. sodium hydroxide $(0.5 \mathrm{~mL})$. The reaction mixture was stirred at $-30{ }^{\circ} \mathrm{C}$ for 3 $\mathrm{h}$, then at $25^{\circ} \mathrm{C}$ for $2 \mathrm{~h}$. The solvent was evaporated, the residual solid was suspended in water $(1$ $\mathrm{mL}$ ) and extracted with dichloromethane. The organic solvent was removed under reduced pressure and the crude solid was purified by flash chromatography (hexane/dichloromethane, 0 to $100 \%$ ). The first fractions contained 4-chloro- $N, N$-dimethyl-3-nitrobenzenesulfonamide (2c) as a light yellow crystalline solid $(1.86 \mathrm{~g}, 60 \%)$ followed by fractions containing 4-dimethylamino- $N, N$-dimethyl-3-nitrobenzenesulfonamide (5) as a yellow crystalline solid (308 $\mathrm{mg}, 20 \%$ ). 2c: $\mathrm{mp} 98-100{ }^{\circ} \mathrm{C} . \mathrm{R}_{f}=0.40$ (dichloromethane/hexane, 2:1). IR (KBr): $\tilde{v}$ 1531, 1360, 1340, $1154,1047,960,777,711 \mathrm{~cm}^{-1} .{ }^{1} \mathrm{H}$ NMR $\left(300 \mathrm{MHz}, \mathrm{CDCl}_{3}\right): \delta 2.80\left(\mathrm{~s}, 6 \mathrm{H}, \mathrm{NMe}_{2}\right), 7.76\left(\mathrm{~d},{ }^{3} J=\right.$ $8.5 \mathrm{~Hz}, 1 \mathrm{H}, \mathrm{H}-5) 7.90$ (dd, $\left.{ }^{3} J=8.5 \mathrm{~Hz},{ }^{4} J=2.3 \mathrm{~Hz}, 1 \mathrm{H}, \mathrm{H}-6\right), 8.25\left(\mathrm{~d},{ }^{4} J=2.0 \mathrm{~Hz}, 1 \mathrm{H}, \mathrm{H}-2\right) .{ }^{13} \mathrm{C}$ NMR (125 MHz, $\mathrm{CDCl}_{3}$ ): $\delta 38.0$ (2C), 124.9, 131.7, 131.9, 133.2, 136.6, 148.2. MS (ESI) $\mathrm{m} / \mathrm{z}$ (\%) $265.0(25)[\mathrm{M}+\mathrm{H}]^{+}$.

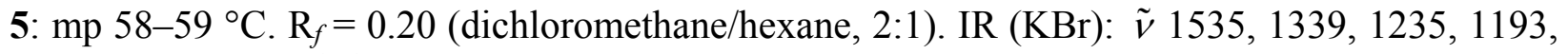
$1043,887,669 \mathrm{~cm}^{-1} .{ }^{1} \mathrm{H}$ NMR $\left(400 \mathrm{MHz}, \mathrm{CDCl}_{3}\right): \delta 2.75\left[\mathrm{~s}, 6 \mathrm{H}, \mathrm{NMe}_{2}\right], 3.05\left(\mathrm{~s}, 6 \mathrm{H}, \mathrm{NMe}_{2}\right), 7.1$ $\left(\mathrm{d},{ }^{3} J=9.0 \mathrm{~Hz}, 1 \mathrm{H}, \mathrm{H}-5\right), 7.75$ (dd, $\left.{ }^{3} J=9.0 \mathrm{~Hz},{ }^{4} J=2.3 \mathrm{~Hz}, 1 \mathrm{H}, \mathrm{H}-6\right), 8.2\left(\mathrm{~d},{ }^{4} J=2.3 \mathrm{~Hz}, 1 \mathrm{H}\right.$, H-2). ${ }^{13} \mathrm{C}$ NMR (100 MHz, $\mathrm{CDCl}_{3}$ ): $\delta 37.9$ (2C), $\delta 42.2(2 \mathrm{C}), 117.3(2 \mathrm{C}), 127.5(2 \mathrm{C}), 131.8$ (2C). Anal. calcd. for $\mathrm{C}_{10} \mathrm{H}_{15} \mathrm{~N}_{3} \mathrm{O}_{4} \mathrm{~S}$ (273.31): C, 43.95; H, 5.53; N, 15.37; S, 11.73. Found: C, 43.93; H, 5.57; N, 15.35; S, 11.71 . 
$\boldsymbol{N}, \boldsymbol{N}$-Dimethyl-3-nitro-4-phenylthiobenzenesulfonamide (3c). A solution of 2c (7 g, 26.47 mmol) and benzenethiol $(3 \mathrm{~mL}, 29.20 \mathrm{mmol})$ in freshly distilled THF (32 $\mathrm{mL})$ was added dropwise to a suspension of $\mathrm{NaH}(2 \mathrm{~g}, 83.30 \mathrm{mmol})$ in freshly distilled THF $(25 \mathrm{~mL})$. After the reaction mixture has been stirred at room temperature for $20 \mathrm{~h}$, it was slowly poured on ice. The resulting crude product was purified by column chromatography (dichloromethane/hexane, 3:1) to afford a yellow solid 3c $\left(8.4 \mathrm{~g}, 94 \%\right.$ ); mp $123-125{ }^{\circ} \mathrm{C} . \mathrm{R}_{f}=0.50$ (dichloromethane/hexane 3:1). IR (KBr): $\tilde{v} 1506,1343,1330,1161,955,776,712 \mathrm{~cm}^{-1} .{ }^{1} \mathrm{H}$ NMR $\left(500 \mathrm{MHz}, \mathrm{CDCl}_{3}\right): \delta$ $2.75\left(\mathrm{~s}, 6 \mathrm{H}, \mathrm{NMe}_{2}\right), 7.00\left(\mathrm{~d},{ }^{3} J=8.5 \mathrm{~Hz}, 1 \mathrm{H}, \mathrm{H}-5\right), 7.58-7.63\left(\mathrm{~m}, 5 \mathrm{H}, \mathrm{H}-2^{\prime}, 3^{\prime}, 4^{\prime}, 5^{\prime}, 6^{\prime}\right), 7.68$ $\left(\mathrm{dd},{ }^{3} J=8.7 \mathrm{~Hz},{ }^{4} J=2.1 \mathrm{~Hz}, 1 \mathrm{H}, \mathrm{H}-6\right), 8.63$ (d, $\left.{ }^{4} \mathrm{~J}=1.9 \mathrm{~Hz}, 1 \mathrm{H}, \mathrm{H}-2\right) .{ }^{13} \mathrm{C}$ NMR $(125 \mathrm{MHz}$, $\left.\mathrm{CDCl}_{3}\right): \delta 37.7$ (2C), 125.0, 128.6, 129.2, 130.4 (2C), 130.7, 131.1, 132.8, 135.9 (2C), 144.0, 145.5. MS (APCI/MeOH): $m / z(\%) 339.1(100)[\mathrm{M}+\mathrm{H}]^{+}$.

$\mathbf{N}, \mathbf{N}$-Dimethyl-10H-phenothiazine-2-sulfonamide (4c). A solution of 3c (1.88 g, $5.56 \mathrm{mmol})$, peroxide-free cumene $(54 \mathrm{~mL})$ and triethylphosphite $(3.7 \mathrm{~mL}, 21.58 \mathrm{mmol})$ was refluxed under nitrogen for $10 \mathrm{~h}$ to give $4 \mathrm{c}$ as a yellow solid (1.12 g, 66\%). This product was immediately used without purification for the next step. An analytical sample of $4 \mathbf{c}$ was purified by column chromatography (hexane/dichloromethane, 0 to $100 \%$ ); mp $154-157^{\circ} \mathrm{C}$. $\mathrm{R}_{f}=0.30$ (dichloromethane/hexane 3:1). IR (KBr): $\tilde{v} 3345,1458,1332,1159,960,758 \mathrm{~cm}^{-1}$. ${ }^{1} \mathrm{H}$ NMR (500 MHz, $\left.\mathrm{CDCl}_{3}\right): \delta 2.75\left(\mathrm{~s}, 6 \mathrm{H}, \mathrm{NMe}_{2}\right), 6.40(\mathrm{~s}, \mathrm{NH}, 1 \mathrm{H}), 6.60\left(\mathrm{~d},{ }^{3} J=8.0 \mathrm{~Hz}, 1 \mathrm{H}, \mathrm{H}-8\right), 6.85\left(\mathrm{t},{ }^{3} J=8.0\right.$ $\mathrm{Hz}, 1 \mathrm{H}, \mathrm{H}-6), 6.95$ (d, $\left.{ }^{3} \mathrm{~J}=8.0 \mathrm{~Hz}, 1 \mathrm{H}, \mathrm{H}-5\right), 7.05$ (m, 3H, H-1, H-3, H- 7), 7.15 (d, ${ }^{3} \mathrm{~J}=8.0 \mathrm{~Hz}$ ,1H, H-4). ${ }^{13} \mathrm{C}$ NMR (100 MHz, $\left.\mathrm{CDCl}_{3}\right): \delta 38.0$ (2C), 112.7, 115.0, 116.6, 121.2, 123.2, 125.0, 126.7, 126.9, 128.0, 133.8, 140.6, 142.5. MS (APCI/MeOH): $m / z$ (\%) 307.0 (100) $[\mathrm{M}+\mathrm{H}]^{+}$.

$\mathrm{N}$-Acetyl-2-(piperidin-4-yl)ethanol (9). A mixture of malonic acid (0.26 g, $2.49 \mathrm{mmol}), 2$ (piperidin-4-yl)ethanol $(6 ; 0.22 \mathrm{~mL}, 1.62 \mathrm{mmol})$ and anhydrous $\mathrm{Na}_{2} \mathrm{SO}_{4}$ was heated to $110{ }^{\circ} \mathrm{C}$ for $6 \mathrm{~h}$. Thereafter, the mixture was cooled, washed with water and extracted with ethyl acetate $(3 \times 10 \mathrm{~mL})$. The solvent was removed under reduced pressure and the residual oil was purified by preparative TLC (dichloromethane/methanol, 4:1). Compound 9 was obtained as oil (163 mg, $40 \%$ ). $\mathrm{R}_{f}=0.20$ (dichloromethane/methanol, 4:1). IR (film): $\tilde{v}$ 3400, 2927, 1734, 1620, 1451, $1272 \mathrm{~cm}^{-1}$. ${ }^{1} \mathrm{H}$ NMR (400 MHz, $\left.\mathrm{CDCl}_{3}\right): \delta 1.10-1.20(\mathrm{~m}, 2 \mathrm{H}, \mathrm{CHH}), 1.50-1.60$ (q, $2 \mathrm{H},{ }^{3} J=8$ $\left.\mathrm{Hz}, \mathrm{CH}_{2}\right), 1.70-1.85(\mathrm{~m}, 3 \mathrm{H}), 2.00(\mathrm{bs}, 1 \mathrm{H}, \mathrm{OH}), 2.10\left(\mathrm{~s}, 3 \mathrm{H}, \mathrm{CH}_{3}\right), 2.50-2.60\left(\mathrm{td},{ }^{2} \mathrm{~J}=8.0 \mathrm{~Hz}\right.$, $\left.{ }^{3} J=8.0 \mathrm{~Hz},{ }^{3} J=2.0 \mathrm{~Hz}, 1 \mathrm{H}, \mathrm{CHH}\right), 3.00-3.10\left(\mathrm{td},{ }^{2} J=8.0 \mathrm{~Hz},{ }^{3} J=8.0 \mathrm{~Hz},{ }^{3} \mathrm{~J}=2.0 \mathrm{~Hz}, 1 \mathrm{H}\right.$, $\mathrm{CHH}), 3.70-3.75\left(\mathrm{t},{ }^{3} \mathrm{~J}=8.0 \mathrm{~Hz}, 2 \mathrm{H}, \mathrm{CH}_{2} \mathrm{OH}\right), 3.75-3.85(\mathrm{~m}, 1 \mathrm{H}, \mathrm{CHH}), 4.55-4.65(\mathrm{~m}, 1 \mathrm{H}$, $\mathrm{CHH}) .{ }^{13} \mathrm{C} \mathrm{NMR}\left(100 \mathrm{MHz}, \mathrm{CDCl}_{3}\right): \delta 21.5,31.8,32.5,32.6,39.1,41.8,46.7,60.2,168.9$. Anal. calcd. for $\mathrm{C}_{9} \mathrm{H}_{17} \mathrm{NO}_{2}$ (171.07): C, 63.13; H, 10.01; N, 8.18. Found: C, 63.10; H: 10.02; N, 8.20.

10-(3-Chloropropionyl)-10H-phenothiazine (10a). 3-Chloropropionyl chloride (2.64 mL, $27.60 \mathrm{mmol})$ was added to a stirred solution of $4 \mathbf{a}(2.22 \mathrm{~g}, 11.50 \mathrm{mmol})$ in dry benzene $(16 \mathrm{~mL})$ under a nitrogen atmosphere. The solution was heated to $130^{\circ} \mathrm{C}$ for $6 \mathrm{~h}$. The solvent was removed under reduced pressure and the residue was suspended in dichloromethane, washed with $\mathrm{NaOH}(10 \%)$, dried over anhydrous $\mathrm{MgSO}_{4}$, and then the solvent was removed under reduced pressure. The crude grey solid was recrystallized to afford a white solid 10a (1.99 g, $62 \%$ ); $\mathrm{mp} 127-130{ }^{\circ} \mathrm{C}$ (dichloromethane). $\mathrm{R}_{f}=0.5$ (dichloromethane/hexane, $\left.4: 1\right) . \mathrm{IR}(\mathrm{KBr}$ ): $\tilde{v}$ 
1677, 1589, 1461, 1369, $1257 \mathrm{~cm}^{-1} .{ }^{1} \mathrm{H}$ NMR (300 MHz, $\left.\mathrm{CDCl}_{3}\right): \delta 2.80\left(\mathrm{bs}, 2 \mathrm{H}, \mathrm{CH}_{2} \mathrm{CO}\right), 3.80$ (m, 2H, $\left.\mathrm{CH}_{2} \mathrm{Cl}\right), 7.25-7.30$ (t, $\left.{ }^{3} \mathrm{~J}=7.7 \mathrm{~Hz}, 2 \mathrm{H}, \mathrm{H}-3, \mathrm{H}-7\right), 7.35-7.40$ (t, ${ }^{3} J=7.4 \mathrm{~Hz}, 2 \mathrm{H}, \mathrm{H}-2, \mathrm{H}-$ 8), 7.45-7.50 (d, $\left.{ }^{3} J=7.7 \mathrm{~Hz}, 2 \mathrm{H}, \mathrm{Ar}\right), 7.55-7.50$ (bs, 2H, Ar). ${ }^{13} \mathrm{C} \mathrm{NMR}\left(100 \mathrm{MHz}, \mathrm{CDCl}_{3}\right): \delta$ 37.3, 39.6, 126.7, 126.8, 127.1 (3C), 127.2, 127.8, 128.1, 128.4 (2C), 129.5, 138.2, 168.8.

A mixture of 3-chloropropionyl chloride $(2.64 \mathrm{~mL}, 27.60 \mathrm{mmol})$ and $4 \mathrm{a}(2.22 \mathrm{~g}, 11.50 \mathrm{mmol})$ was irradiated in a microwave oven at $700 \mathrm{~W}$ for $7 \mathrm{~min}$ to yield 10a $(3.05 \mathrm{~g}, 95 \%)$.

10-[3-[4-(2-Hydroxyethyl)piperidin-1-yl]propionyl]-10H-phenotiazine (11a). Anhydrous $\mathrm{K}_{2} \mathrm{CO}_{3}(552 \mathrm{mg}, 3.78 \mathrm{mmol})$ and 2-(piperidin-4-yl)ethanol (6; $\left.129 \mathrm{mg}, 1.00 \mathrm{mmol}\right)$ were added to a solution of 10a $(0.394 \mathrm{~g}, 0.99 \mathrm{mmol})$ in dry and freshly distilled DMF $(6 \mathrm{~mL})$. The resulting mixture was refluxed for $2 \mathrm{~h}$. The solvent was evaporated and the residual dark green oil was purified by preparative TLC (dichloromethane/hexane 1:9) to yield 11a as a green oil (189 mg, 50\%). $\mathrm{R}_{f}=0.25$ (dichloromethane/hexane, 1:9). IR (film): $\tilde{v} 3330,2925,1671,1464,1307 \mathrm{~cm}^{-1}$. ${ }^{1} \mathrm{H}$ NMR $\left(300 \mathrm{MHz}, \mathrm{CDCl}_{3}\right): \delta 1.05(\mathrm{~m}, 2 \mathrm{H}, \mathrm{CHH}), 1.30\left(\mathrm{~m}, 3 \mathrm{H},-\mathrm{CH}_{2} \mathrm{CH}\right), 1.50\left(\mathrm{~d},{ }^{3} \mathrm{~J}=8.0 \mathrm{~Hz}\right.$, $\left.2 \mathrm{H}, \mathrm{CH}_{2}\right), 1.70\left(\mathrm{~m}, 2 \mathrm{H}, \mathrm{CH}_{2}\right), 1.95\left(\mathrm{~m}, 2 \mathrm{H}, \mathrm{CH}_{2}\right), 2.45\left(\mathrm{~m}, 2 \mathrm{H}, \mathrm{CH}_{2}\right), 2.55\left(\mathrm{~m}, 4 \mathrm{H}, 2 \mathrm{CH}_{2}\right), 2.70$ (bs, $1 \mathrm{H}, \mathrm{OH}), 3.45$ (t, $\left.{ }^{3} J=6.3 \mathrm{~Hz}, 2 \mathrm{H}, \mathrm{CH}_{2} \mathrm{OH}\right), 6.55\left(\mathrm{~d},{ }^{3} \mathrm{~J}=7.2 \mathrm{~Hz}, 1 \mathrm{H}, \mathrm{Ar}\right), 6.83\left(\mathrm{t},{ }^{3} J=7.5\right.$ $\mathrm{Hz}, 1 \mathrm{H}, \mathrm{Ar}), 7.00\left(\mathrm{~d},{ }^{3} \mathrm{~J}=6.9 \mathrm{~Hz}, 1 \mathrm{H}, \mathrm{Ar}\right), 7.20\left(\mathrm{t},{ }^{3} J=7.7 \mathrm{~Hz} 1 \mathrm{H}, \mathrm{Ar}\right), 7.30\left(\mathrm{t},{ }^{3} J=6.9 \mathrm{~Hz}, 2 \mathrm{H}\right.$, Ar), $7.45\left(\mathrm{~d},{ }^{3} J=7.7 \mathrm{~Hz}, 1 \mathrm{H}, \mathrm{Ar}\right), 7.55\left(\mathrm{~d},{ }^{3} J=7.7 \mathrm{~Hz}, 1 \mathrm{H}, \mathrm{Ar}\right) .{ }^{13} \mathrm{C}$ NMR $\left(75 \mathrm{MHz}, \mathrm{CDCl}_{3}\right): \delta$ 30.0, 32.1, 39.5, 53.1, 54.0, 55.2, 58.5, 60.1, 61, 114.2 (2C), 118.8, 123.3 (2C), 127.0, 128.1 (2C), 128.8 (2C), 139.1, 142.0, 171.2. Anal. calcd. for $\mathrm{C}_{22} \mathrm{H}_{26} \mathrm{~N}_{2} \mathrm{O}_{2} \mathrm{~S}$ (382.26): C, 69.08; H, 6.85; N, 7.32; S, 8.38. Found: C, 69.10; H, 6.89; N, 7.30; S, 8.35.

10-(3-Chloropropionyl)- $\mathbf{N}, \boldsymbol{N}$-dimethyl-10H-phenothiazine-2-sulfonamide (10c). As for 10a, the reaction of 3-chloropropionyl chloride $(2.64 \mathrm{~mL}, 27.60 \mathrm{mmol})$ and $4 \mathrm{c}(2.22 \mathrm{~g}, 7.25 \mathrm{mmol})$ in dry benzene $(16 \mathrm{~mL})$ gave a crude yellowish-white solid that was purified by recrystallization to afford a white solid 10c (1.78 g, 62\%); mp 195-198 ${ }^{\circ} \mathrm{C}$ (dichloromethane). $\mathrm{R}_{f}=0.25$ (dichloromethane/hexane, 3:1). IR (KBr): $\tilde{v} 1681,1334,1158,780,715,610 \mathrm{~cm}^{-1}$. ${ }^{1} \mathrm{H}$ NMR $\left(500 \mathrm{MHz}, \mathrm{CDCl}_{3}\right): \delta 2.75$ (s, 6H, NMe $), 2.80$ (bs, 1H, CHHCO), 3.10 (bs, 1H, CHHCO), 3.85 $\left(\mathrm{m}, 2 \mathrm{H}, \mathrm{CH}_{2} \mathrm{Cl}\right), 7.29\left(\mathrm{td},{ }^{3} J=7.8 \mathrm{~Hz},{ }^{4} \mathrm{~J}=1.4 \mathrm{~Hz}, 1 \mathrm{H}, \mathrm{H}-6\right), 7.37\left(\mathrm{td},{ }^{3} J=7.6 \mathrm{~Hz},{ }^{4} \mathrm{~J}=1.4 \mathrm{~Hz}\right.$, $1 \mathrm{H}, \mathrm{H}-7), 7.50$ (m, 2H, H-5, H-8), 7.55 (d, $\left.{ }^{3} J=8.0 \mathrm{~Hz}, 1 \mathrm{H}, \mathrm{H}-4\right), 7.61\left(\mathrm{dd},{ }^{3} J=8.2 \mathrm{~Hz},{ }^{4} J=1.8\right.$ $\mathrm{Hz}, 1 \mathrm{H}, \mathrm{H}-3), 7.98$ (s, 1H, H-1). ${ }^{13} \mathrm{C}$ NMR (125 MHz, $\left.\mathrm{CDCl}_{3}\right): \delta 37.4(2 \mathrm{C}), 38.2,39.5,126.1$ (2C), 127.2, $128.0(2 \mathrm{C}), 128.1$ (2C), 128.2, 128.7 (2C), 137.6, 138.6, 168.9. MS (APCI/ MeOH): $\mathrm{m} / \mathrm{z}(\%) 397.1(50)[\mathrm{M}+\mathrm{H})]^{+}$.

A mixture of 3-chloropropionyl chloride $(2.64 \mathrm{~mL}, 27.6 \mathrm{mmol})$ and $4 \mathrm{c}(2.22 \mathrm{~g}, 7.25 \mathrm{mmol})$ upon irradiation in a microwave oven at $700 \mathrm{~W}$ for $7 \mathrm{~min}$ gave $10 \mathrm{c}(2.81 \mathrm{~g}, 98 \%)$.

10-[3-[4-(2-Hydroxyethyl)-piperidin-1-yl]propionyl]- $\mathbf{N}, \mathbf{N}$-dimethyl-10H-phenotiazine-2-

sulfonamide (11c). Anhydrous $\mathrm{K}_{2} \mathrm{CO}_{3}(552 \mathrm{mg}, 3.78 \mathrm{mmol})$ and 2-(piperidin-4-yl)ethanol (6; $129 \mathrm{mg}, 1.00 \mathrm{mmol})$ were added to a solution of $10 \mathrm{c}(0.382 \mathrm{~g}, 1.00 \mathrm{mmol})$ in dry and freshly distilled DMF $(6 \mathrm{~mL})$. The resulting mixture was irradiated in a domestic microwave oven at 150W for $2 \mathrm{~min}$. The solvent was removed under reduced pressure, and the oily residue was purified by preparative TLC (dichloromethane/hexane/ethanol, 10:1:0.2). Compound 11c was obtained as oil (0.396 g, 81\%). $\mathrm{R}_{f}=0.2$ (dichloromethane/hexane/ethanol, 10:1:0.2). IR (film): $\tilde{v}$ 
1681, 1334, 1158, 780, 715, $610 \mathrm{~cm}^{-1} .{ }^{1} \mathrm{H}$ NMR $\left(500 \mathrm{MHz}, \mathrm{CDCl}_{3}\right): \delta 1.20-1.50(\mathrm{~m}, 6 \mathrm{H}), 1.60$ (m, $2 \mathrm{H}), 2.0(\mathrm{~m}, 2 \mathrm{H}), 2.50(\mathrm{bs}, 1 \mathrm{H}, \mathrm{OH}), 2.62(\mathrm{~m}, 2 \mathrm{H}), 2.75\left(\mathrm{~s}, 6 \mathrm{H}, \mathrm{NMe}_{2}\right), 2.80(\mathrm{~s}, 1 \mathrm{H}), 3.10$ (bs, 2H), 3.80 (t, $\left.{ }^{3} J=7.0,2 \mathrm{H}, \mathrm{CH}_{2} \mathrm{OH}\right), 7.29\left(\mathrm{t},{ }^{3} J=7.8 \mathrm{~Hz},{ }^{4} J=1.4 \mathrm{~Hz}, 1 \mathrm{H}, \mathrm{H}-6\right), 7.37$ (td, ${ }^{3} J=$ $\left.7.6 \mathrm{~Hz},{ }^{4} J=1.4 \mathrm{~Hz}, 1 \mathrm{H}, \mathrm{H}-7\right), 7.50$ (m, 2H, H-5, H-8), 7.55 (d, $\left.{ }^{3} J=8.0 \mathrm{~Hz}, 1 \mathrm{H}, \mathrm{H}-4\right), 7.61$ (dd, ${ }^{3} J$ $\left.=8.2 \mathrm{~Hz},{ }^{4} J=1.8 \mathrm{~Hz}, 1 \mathrm{H}, \mathrm{H}-3\right), 8.00(\mathrm{~s}, 1 \mathrm{H}, \mathrm{H}-1) .{ }^{13} \mathrm{C} \mathrm{NMR}\left(125 \mathrm{MHz}, \mathrm{CDCl}_{3}\right): \delta 29.7,32.1$, $32.8,37.9$ (2C), 39.3, 46.1, 47.2, 54.0, 59.8, 60.25, 122.7, 123.3, 125.9, 127.9, 128.1, 128.4, 129.8, 131.4, 134.2, 136.0, 137.1, 138.6. MS (APCI/MeOH): $m / z(\%) 490.2(100)[\mathrm{M}+\mathrm{H}]^{+}$. Anal. calcd. for $\mathrm{C}_{24} \mathrm{H}_{31} \mathrm{~N}_{3} \mathrm{O}_{4} \mathrm{~S}_{2}$ (489.32): C, 58.87; H, 6.38; N, 8.58; S, 13.10. Found: C, 58.85; H, $6.39 ; \mathrm{N}, 6.40 ; \mathrm{S}, 13.00$.

\section{0-[3-[4-(2-Hydroxyethyl)piperidin-1-yl]propyl]- $N, N$-dimethyl-10H-phenothiazine-2-}

sulfonamide (Pipothiazine, 1). Trifluoroborane etherate $(1 \mathrm{~mL}, 0.79 \mathrm{mmol})$ was added dropwise to a stirred mixture of $\mathrm{NaBH}_{4}(294 \mathrm{mg}, 7.77 \mathrm{mmol})$ in diglyme $(15 \mathrm{~mL})$ at room temperature. The borane gas generated was carried over with nitrogen and bubbled into a solution of $11 \mathrm{c}(81 \mathrm{mg}, 0.16 \mathrm{mmol})$ in dry THF $(2 \mathrm{~mL})$. The mixture was stirred at room temperature for $48 \mathrm{~h}$. Excess of borane was eliminated by careful addition of diluted $\mathrm{HCl}$ until $\mathrm{pH}=6$ was reached. The organic phase was washed with water and the solvent was removed. The crude product was purified by preparative TLC (dichloromethane/methanol, 6:1) to afford a yellow oil 1 (57 mg, 72\%) that was compared with an authentic sample (NMR, IR). $\mathrm{R}_{f}=0.4$ (dichloromethane/ethanol, 8:1). IR (film): $\tilde{v} 3369,2917,2849,1455,1408,1342,1162,731,621$ $\mathrm{cm}^{-1} .{ }^{1} \mathrm{H}$ NMR $\left(500 \mathrm{MHz}, \mathrm{CDCl}_{3}\right): \delta 1.30(\mathrm{~m}, 3 \mathrm{H}), 1.51(\mathrm{~m}, 2 \mathrm{H}), 1.68(\mathrm{~m}, 2 \mathrm{H}), 2.0(\mathrm{~m}, 4 \mathrm{H})$, $2.45\left(\mathrm{t},{ }^{3} J=7.2 \mathrm{~Hz}, 2 \mathrm{H}, \mathrm{NCH}_{2}\right), 2.70\left(\mathrm{~s}, 6 \mathrm{H}, \mathrm{NMe}_{2}\right), 2.90(\mathrm{~m}, 2 \mathrm{H}), 3.68(\mathrm{t}, J=6.5 \mathrm{~Hz}, 2 \mathrm{H}$, $\left.\mathrm{NCH}_{2}\right), 3.95\left(\mathrm{t}, J=6.9 \mathrm{~Hz}, 2 \mathrm{H}, \mathrm{CH}_{2} \mathrm{OH}\right), 6.95(\mathrm{~m}, 2 \mathrm{H}, \mathrm{Ar}), 7.11\left(\mathrm{dd},{ }^{3} J=7.7 \mathrm{~Hz},{ }^{4} J=1.5 \mathrm{~Hz}\right.$, $1 \mathrm{H}, \mathrm{Ar}), 7.14-7.30$ (m, 4H, Ar). ${ }^{13} \mathrm{C}$ NMR $\left(125 \mathrm{MHz}, \mathrm{CDCl}_{3}\right): \delta 24.1,29.6,32.0,32.1,37.9$ (2C), 39.2, 45.5, 53.8 (2C), 55.6, 60.3, 113.9, 116.1, 121.2, 123.2, 123.5, 127.3, 127.4, 127.7, $131.4,134.3,143.9,145.8$.

\section{Acknowledgements}

Financial support of this work by Laboratorio Dosa S.A and University of Buenos Aires is warmly acknowledged.

\section{References}

1. Mosnaim, A.; Ranade, V. Am. J. Ther. 2006, 13, 261.

2. Daversh, S.; McDonald, R. S.; Penwell, A.; Conrad, S.; Darvesh, K. V.; Mataija, D.; Gomez, G.; Caines A.; Walsh, R.; Martin, E. Biorg. Med. Chem. 2005, 13, 211. 
3. (a) Schmidt, M.; Teitge, M.; Castillo M. E.; Brandt, T.; Dobner. B.; Langner, A. Arch. Pharm. (Weinheim) 2008, 341, 624. (b) Bissi, A.; Meli, M.; Gobbi, S.; Rampa, A.; Tolomeo, M.; Dusonchet, L. Bioorg. Med. Chem. 2008, 16, 6474.

4. Jacob, R. M.; Robert, J. G. Ger. Pat. DE 1117584, 1961. Chem. Abstr. 1962, 57, 23257.

5. Yale, H. L. J. Am. Chem. Soc. Mass Spectrom. 1955, 77, 2270.

6. Evans, W. J.; Smiles, S. J. Chem. Soc. 1935, 181, 1263.

7. Smolinski, G.; Feuer, B. I. J. Org. Chem. 1966, 31, 3882.

8. Cadogan J. I. G.; Cameron-Wood, M.; Mackie, R. K., Searle, R. J. J. Chem. Soc.1965, 4831.

9. Cadogan, J. I. G.; Kulik, S., Thomson, C., Todd, M. J. Chem. Soc. (C) 1970, 2437.

10. Moolayil, J. T.; Mathai, G.; Srinivas, R.; Giblin, D.; Russell, A.; Gross, M. L. J. Am. Chem. Soc. Mass Spectrom. 2007, 18, 2204.

11. The Index Merck, 12th Edition 1996, p 1250.

12. Landquist, J. K. In Comprehensive Organic Chemistry; Barton, D. H. R.; Ollis, W. D., Eds.; Pergamon Press: Hill Hall, Oxford, 1979; vol. 4, pp 1102-1107.

13. Plakidin, V. L.; Vostrova, V. N. Zh. Org. Khim. 1982, 18, 342; Chem. Abstr. 1982, 96, 199189.

14. Bonte, J. P.; Debaert, M.; Brunet, C.; Marsau, P.; Cotrait, M. Eur. J. Med. Chem. 1982, 17, 365.

15. Shavyrina, V. V.; Zhuravlev, S. V.; Vikhlyaev, Y. I.; Klygul, T. A.; Slyn'ko, E. I. Khim.Farm. Zh. 1974, 8, 23; Chem. Abstr. 1974, 81, 63569.

16. Leighton, H. J.; Gillies, I. Eur. Pat. Appl. EP 163551, 1985; Chem. Abstr. 1986, 104, 148893.

17. Nazarova, L. S.; Likhosherstov, A. M.; Markova, G. A.; Chichkanov, G. G.; Kaverina, N. V.; Skoldinov, A. P. Khim.-Farm. Zh. 1978, 12, 84; Chem. Abstr. 1978, 89, 190804. 